\title{
Serum Zinc: A Noninvasive Biomarker for the Prediction of Invasive Placentation
}

\author{
${\text { Zainab Abdulameer } \text { Jafaar }^{(\oplus)} \text {, Reshed Zeki Obeid }{ }^{2 *}{ }^{\circledR}, \text { Dina Akeel Salman }}^{1}$
}

\begin{abstract}
Objectives: Abnormally invasive placentation (AIP) is a challenging situation capable of complicating childbirth that has recently arisen, imposing a risk on maternal lives and well-being. The optimal management of this complication requires a valid antenatal diagnostic tool other than Doppler ultrasound (US). Of these, a handful of laboratory markers have been so far used for the antenatal detection of invasive placentation. This study sought to establish whether low maternal serum zinc ( $\mathrm{Zn}$ ) is related to AIP in women with placenta previa.

Materials and Methods: Over a period of six years, a prospective study was performed, including 168 pregnant ladies with placenta previa of whom, 72 cases were diagnosed with placenta accrete (PA) spectrum using Doppler US. The serum Zn was measured in all participants, and each individual was followed up regarding intraoperative and postoperative outcomes.

Results: A significantly lower serum Zn level was found in women having the PA spectrum $(P<0.001)$. At a level of $68 \mu \mathrm{g} / \mathrm{dL}$, serum Zn was $79.2 \%$ sensitive, $95.8 \%$ specific, and had a comparable accuracy $(88.7 \%$ vs. $89.3 \%)$ to color Doppler US in the identification of cases with PAS spectrum among those with placenta previa.

Conclusions: Low maternal serum $\mathrm{Zn}$ level is associated with AIP among placenta previa cases. Thus, more studies are needed in the future to support the use of this technique in the antenatal detection of the PAS.

Keywords: Maternal serum zinc, Placenta accrete spectrum, Placenta previa
\end{abstract}

\section{Introduction}

The management of abnormally invasive placentation (AIP) is one of the most challenging conditions in obstetrics. This condition is a group of disorders, which has more recently been relabeled as placenta accrete spectrum (PAS), that covers all varieties including placenta accrete, placenta increta, and placenta percreta (1). This update was made so as to include both the abnormal adhesion and invasion of trophoblasts into the uterine wall given that the two conditions may coexist alongside one another (2).

Considering the global increase of the cesarean section (CS) rate to $30 \%$ of labors over the last 40 years, the incidence of PAS has increased by more than 10-fold (3). In turn, this has led to the emergence of multiple maternalrelated morbidities, including massive hemorrhage and the need for multiple transfusions, hysterectomy, injury to the abdominal organs, and the need for vascular, bowel, or bladder surgery. In addition, the maternal mortality rate attributed to PAS might be as high as $7 \%(4,5)$.

The PAS management at level III or IV centers by a multidisciplinary team is known to improve maternal morbidity and mortality outcomes (1). To achieve a successful result, the antenatal diagnosis of the condition is of utmost importance to ensure adequate time for referrals to the multidisciplinary team at the time of delivery (4).
Other than the clinical anticipation, which is based on the previous history of CS or uterine surgery, maternal age, and the presence of placenta previa, ultrasound (US) is still preferable to other radiological modalities such as magnetic resonance imaging $(4,6,7)$. In spite of being still recommended as the mainstay for the antenatal diagnosis of PAS, the US neither identifies all cases nor relates to the depth of invasion or histopathological findings $(3,4,7)$.

In addition, its successful use depends on the expertise of the operator, with an interobserver variation in the diagnosis which is entirely possible (1). Many researchers have suggested adding different serum biomarkers in order to improve the detection of AIP, including pregnancy-associated plasma protein-A (PAPP-A), betahuman gonadotropin ( $\beta$-hCG), alpha-fetoprotein, and most recently, cell-free fetal deoxyribonucleic acid and micro ribonucleic acid $(8,9)$.

Zinc $(\mathrm{Zn})$ is an essential trace element previously reported to be related to abnormal fetal growth (10), low birth weight $(11,12)$, and preeclampsia in obstetrics (13-15). As new knowledge, the preterm rupture of the membranes is also associated with reduced umbilical cord and maternal serum $\mathrm{Zn}$ when compared to spontaneous preterm births (15).

Based on previous studies, human trophoblasts and

Received 20 January 2020, Accepted 14 July 2020, Available online 23 October 2020

${ }^{1}$ Department of Obstetrics and Gynecology, College of Medicine, Al-Mustansiriya University, Baghdad, Iraq. ${ }^{2}$ Department of Obstetrics and Gynecology, College of Medicine, Al-Anbar University, Ramadi, Iraq.

*Corresponding Author: Reshed Z. Obeid, Tel: +967705340920, Email: reshedzeki@uoanbar.edu.iq 
Key Messages

- Zinc is an important element in normal placental formation.

- This is the first study that suggest maternal serum zinc as a biomarker for invasive placentation.

cancer cells have demonstrated similar mechanisms by having similar circuitries at the molecular level enabling them to proliferate, migrate, and have the invasive ability $(16,17)$. Regarding the knowledge of the role of $\mathrm{Zn}$ deficiency in the progress of cancer growth and development $(18,19)$, Zn deficiency might be implicated in the process of invasive trophoblastic growth in PAS.

To the best of our knowledge, this study is the first one to employ serum $\mathrm{Zn}$ as a biomarker in the potential detection of PAS cases. The present study attempted to find a new biomarker that is inexpensive, reliable, and readily available to assist in PAS prediction among women with placenta previa. The study further aimed to compare management outcomes between the cases of placenta previa with those of placenta previa with PAS at the tertiary hospital level.

\section{Patients and Methods}

Study Design

A prospective cohort study was carried out in the Department of Obstetrics and Gynecology at Al-Yarmouk Teaching Hospital in Baghdad, Iraq from September 2013 to April 2019.

One hundred sixty-eight patients were enrolled in the current study. They were diagnosed with placenta previa completely covering the os either before admission or coming with antepartum hemorrhage. Doppler US in our hospital was performed by senior US technologists to reveal the diagnosis of placenta previa, and placenta accrete was suggested in some cases. All women in the study were matched in terms of age (30-35 years old) and body mass index (BMI).

\section{Study Population}

Women who met the following inclusion criteria were chosen as study participants:

1. Singleton pregnancy;

2. Gestation age of the fetus between 37 to 40 weeks;

3. Para 1 elective CS with no vaginal bleeding;

4. Diagnosed in the current pregnancy as having placenta previa completely covering the os by Doppler US study.

On the other hand, women who met the following exclusion criteria were removed from the study:

1. History of emergency CS or infection postpartum;

2. Medical illness (e.g., hypertensive disorders including preeclampsia, diabetes mellitus, and liver and renal disease);
3. History of multivitamin intake during pregnancy including $\mathrm{Zn}$;

4. Previous dilation and curettage a scar in the uterus for a reason other than CS (e.g., myomectomy and metroplasty scars).

Participants' medical history was collected, and then several actions were taken, including a general examination, vital signs and BMI measurement, and an obstetric examination. A peripheral blood sample $(5 \mathrm{~mL})$ was also taken from each mother before delivery, and then placed in a sterile container and labeled. Following centrifugation at $3000 \mathrm{rpm}$ for 20 minutes, the supernatant serum was collected in a separate sterile polyethylene container and stored at $-20^{\circ} \mathrm{C}$ until further analysis for measuring the serum $\mathrm{Zn}$.

All patients were sent for confirmatory Doppler US study performed by the same operator, and all surgical operations were done by senior obstetricians in the same department.

After delivery, the study participants were grouped into patients without placenta accreta placenta who delivered completely (group 1) and those with placenta accreta syndrome in which the placenta was not delivered or was delivered partially (group 2).

\section{Statistical Analysis}

The data were analyzed using the Statistical Package for Social Sciences software, version 24 (IBM Corp., Armonk, NY, USA). Categorical data were presented as frequencies and percentages, and their associations were assessed using Pearson's chi-square test. Furthermore, continuous variables were tested for normality and were considered to be non-normal if they showed a skewness of more than 1 . Normally distributed continuous variables were presented with means and standard deviations and the comparisons between the groups were evaluated by the Student's $t$ test (a parametric test). Moreover, non-normally distributed continuous variables were presented with medians and interquartile ranges, and comparisons between the groups were conducted using the Mann-Whitney U test (a nonparametric test). Additionally, multivariate logistic regression was completed to reveal the real risk factors of hysterectomy among women with placenta accrete and to remove confounders. In addition, a receiver operating characteristic curve was used to estimate the predictive value of serum $\mathrm{Zn}$ for the stratification of placenta accreta cases from those with placenta previa. A level of significance was set at $5 \%$.

\section{Results}

A total of 168 pregnant women were included in this study. Among the participants, 96 and 72 cases were included in groups 1 and 2, respectively.

Maternal age, years since last delivery, history of antenatal care, history of antepartum hemorrhage, gestational age, and birth weight presented no significant 
differences between the groups (Table 1). However, the maternal serum levels of $\mathrm{Zn}$ were significantly lower in patients of group 2 (Table 2). Further, a serum $\mathrm{Zn}$ level of $68 \mu \mathrm{g} / \mathrm{dL}$ or below was specifically associated with placenta accrete with a sensitivity rate of $79.2 \%$ and a specificity rate of $95.8 \%$ (Table 2 and Figure 1).

In the comparison of validity while evaluating maternal parameters using the maternal serum levels of $\mathrm{Zn}$ and color Doppler US, serum Zn was more specific and showed a higher positive predictive value compared to Doppler US (95.8\% vs. $88.9 \%$ and $93.4 \%$ vs. $86.5 \%$ ), as well as a comparable level of accuracy to that of Doppler US ( $88.7 \%$ vs. $89.3 \%)$. Nonetheless, serum $\mathrm{Zn}$ demonstrated a lower sensitivity $(79.2 \%$ vs. $88.9 \%)$, the details of which are shown in Table 3 and Figure 2.

At the time of surgery, 96 patients were found to have placenta previa without accrete. Of these, 64 (66.6\%), 12 (12.50\%), 6 (6.25\%), 7 (7.29\%), 4 (4.17\%), cases required an operation simply to remove the placenta, the oversewing of the placental bed, oversewing and packing, required oversewing and B Lynch procedure, and uterine artery ligation, respectively. Eventually, 3 (3.13\%) cases underwent internal iliac artery ligation (Figure 3).

Considering complications arising during surgery, no case of ureteric injury or bladder injury was observed in the placenta previa group (group 1) while $12(16.7 \%)$ and $43(59.7 \%)$ cases were reported in the placenta accrete group (group 2) in this regard. Based on the findings, no patient in the normal placentation group (group 1) died while there were four maternal deaths among the hysterectomy group (group 2). Among these deaths, two patients died in the respiratory care unit (RCU) for acute respiratory distress syndrome, one died as a result of a massive pulmonary embolism, and the fourth mother

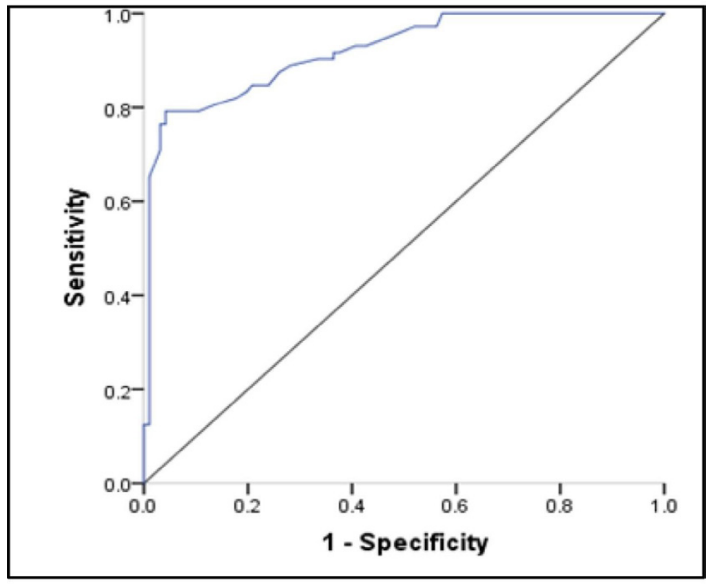

Figure 1. Receiver Operating Characteristic Curve for Estimating the Predictive Value of Serum Zinc for the Stratification of the Cases of Placenta Accreta From Those of Placenta Previa.

died due to acute hypoxia as she had a known case of dilated cardiomyopathy. Total RCU admittance was 3 $(3.1 \%)$ and $36(50 \%)$ in placenta previa and in accreta groups, respectively. Massive blood transfusion, defined as the replacement of more than 10 units of blood within 24 hours (20), was required in $62(86.1 \%)$ of women with placenta accrete, who included a majority of the population of group 2 (Table 4).

The histopathological report of the 72 hysterectomy patient revealed that $32(58 \%), 19(29 \%)$, and $8(13 \%)$ cases experienced the accreta variety, the increta variety, and the percreta variety of PAS, respectively (Figure 4).

\section{Discussion}

As patients with PAS are at the risk of a number of

Table 1. General and Obstetrical Characteristics of Patients

\begin{tabular}{|c|c|c|c|}
\hline \multirow{2}{*}{ Parameter } & Placenta Previa Without Accreta $(n=96)$ & Placenta Previa With Accreta $(n=72)$ & \multirow{2}{*}{$P$ Value } \\
\hline & \multicolumn{2}{|c|}{ Mean \pm SD } & \\
\hline Maternal age (y) & $35.4 \pm 5$ & $35.4 \pm 4.7$ & 0.5 \\
\hline Years since last delivery & $2 \pm 0.7$ & $2.1 \pm 0.9$ & 0.403 \\
\hline Gestational age (wk) & $38.9 \pm 1.8$ & $38.8 \pm 1.2$ & 0.688 \\
\hline \multirow[t]{2}{*}{ Birth weight $(\mathrm{kg})$} & $3.2 \pm 0.6$ & $3.3 \pm 0.7$ & 0.819 \\
\hline & & & \\
\hline History of antenatal care & $36(37.5)$ & $34(47.2)$ & 0.206 \\
\hline History of antepartum hemorrhage & $68(70.8)$ & $44(61.1)$ & 0.186 \\
\hline
\end{tabular}

Note. *Significance at 0.05; SD: syandard deviation; n: Number.

Table 2. The Predictive Value of Serum Zinc Among the Study Group

\begin{tabular}{|c|c|c|c|}
\hline Parameter & Placenta Previa Without Accreta $(n=96)$ & Placenta Previa With Accreta $(n=72)$ Mean \pm SD & $P$ Value \\
\hline Serum zinc $(\mu g / d L)$ & $87.7 \pm 13.6$ & $57.6 \pm 13.5$ & $<0.001^{*}$ \\
\hline The predictive cutoff value of serum $\mathrm{Zn}$ & & $68 \mu \mathrm{g} / \mathrm{dL}$ & \\
\hline
\end{tabular}

Note. *Significance at 0.05; SD: standard deviation 
Table 3. Validity Parameters of the Color Doppler US Diagnosis of Placenta Previa Accreta Among the Study Groups

\begin{tabular}{|c|c|c|c|}
\hline $\begin{array}{l}\text { Placenta Accreta Diagnosis } \\
\text { By Color Doppler US }\end{array}$ & $\begin{array}{c}\text { Placenta Previa } \\
\text { With Accreta }(n=72)\end{array}$ & $\begin{array}{c}\text { Placenta Previa } \\
\text { Without Accreta }(n=96)\end{array}$ & Total \\
\hline Positive & 64 & 10 & 74 \\
\hline Negative & 8 & 86 & 94 \\
\hline Total & 72 & 96 & 168 \\
\hline
\end{tabular}

$\mathrm{SN}=88.9 \%, \mathrm{SP}=89.6 \%, \mathrm{PPV}=86.5 \%, \mathrm{NPV}=91.5 \%$, Accuracy $=89.3 \%$

Note. US: Ultrasound; SN, sensitivity; SP, specificity; PPV, positive predictive value; NPV, negative predictive value.

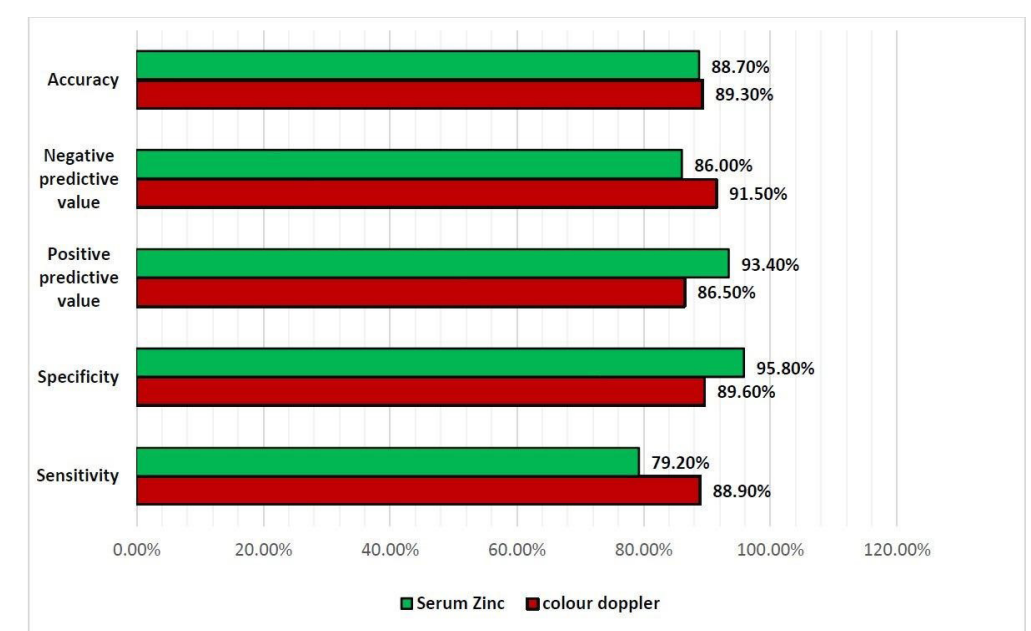

Figure 2. Comparison of the Validity Parameters of the Color Doppler US With Those of Maternal Serum Zinc. Note. US: ultrasound.

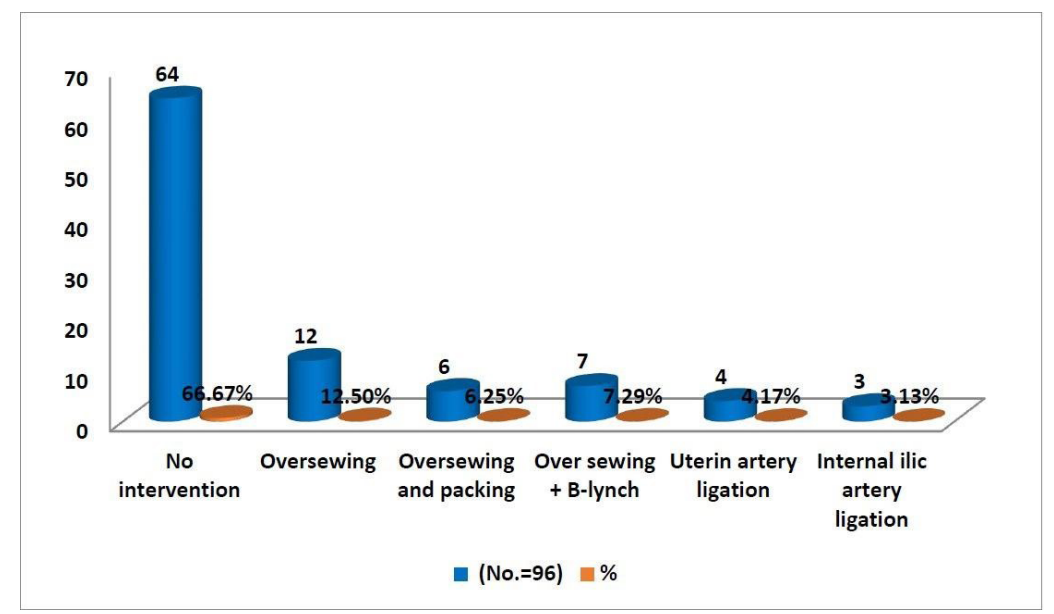

Figure 3. Operative Management of Cases With Placenta Previa Without Accrete.

morbidities and even maternal mortality, antenatal diagnosis remains crucial to ensure enough time for referrals to specialized centers for planned delivery (21). Besides Doppler US, variations in levels of a handful of serum biomarkers were added to enhance the antenatal identification of PAS. Among these, the first and second trimester markers of aneuploidy such as PAPP-A, $\beta$-hCG, and maternal serum alpha-fetoprotein (MSAF) and other markers including micro ribonucleic acid (miRNA) were suggested in this study.

Büke et al studied the first trimester markers and found that both PAPP-A (1.20 vs. 0.865, $P=0.045)$ and f $\beta$-hCG (1.42 vs. $0.93, P=0.042)$ were significantly lower among women with PAS compared to those with placenta previa alone (21). These findings were also reported by Thompson et al (6) and Desai et al (22). Furthermore, Lyell et al concluded that both low PAPP-A and elevated MSAFP (maternal serum alpha fetoprotein) were related 
Table 4. Intraoperative Complications of the Study Groups

\begin{tabular}{|c|c|c|c|c|}
\hline Complications & Placenta Previa Without Accreta $(n=96)$ & $\%$ & Placenta Previa With Accreta $(n=72)$ & $\%$ \\
\hline Ureteric injury & - & - & 12 & 16.7 \\
\hline Bladder injury & - & - & 43 & 59.7 \\
\hline Maternal death & - & - & 4 & 5.6 \\
\hline RCU admission & 3 & 3.1 & 36 & 50 \\
\hline Massive blood transfusion & 6 & 6.3 & 62 & 86.1 \\
\hline
\end{tabular}

Note. RCU: respiratory care unit.

to AIP. In addition, a three-fold increased risk for placenta accreta was independently observed among women with the AFP levels of greater than 1.79 multiples of the median (MoM) or in the 95th percentile (7). The same findings were also revealed by Oztas et al who demonstrated that at a cutoff value of 1.25 MoM, elevated MSAF can predict the risk of hysterectomy in women with placenta previa for PAS with a sensitivity rate of $85.94 \%$ and specificity rate of $71.43 \%$ (23). However, the main limitation of the above-mentioned studies was their retrospective nature and typically small population size.

More recently, in a prospective study, Adami et al proposed maternal serum miRNA as a new biomarker for the detection of placenta accrete and found that women with PAS have a significantly higher total percentage of miRNA in their serum analysis as compared with those presenting normal placentation (9).

In this work, maternal serum $\mathrm{Zn}$ was hypothesized as a feasible biomarker for the detection of PAS among cases of placenta previa based on the understanding of the pathophysiology, molecular, acellular, and the genetic pathologies of PAS. Extensive neovascularization in PAS involves the enhancement of angiogenesis and the instigation of the vascular endothelial growth factor related to a dietary deficiency of $\mathrm{Zn}(24,25)$. In addition, abnormally invasive trophoblasts were associated with the infiltration of subpopulation groups of leukocytes, an increase in endometrial forkhead box P3+ (FoxP3+) regulatory T-cells, a slight increase in the cluster of differentiation 25+ (CD25+) T-cells, and finally, a

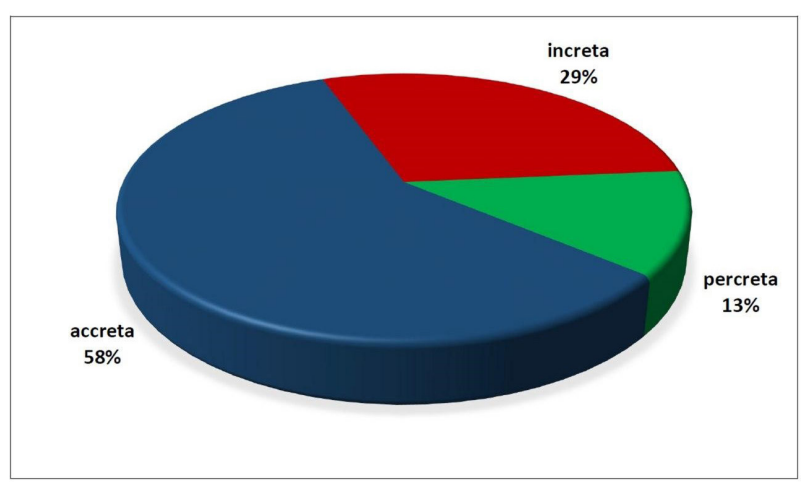

Figure 4. Distribution of the Histopathological Reports of Hysterectomy Patients. significant reduction in decidual natural killer cells (dNKC) as compared with normal pregnancies (26). Interestingly, the functions of CD25+ and FoxP3+ were demonstrated to be suppressed by $\mathrm{Zn}$ supplements (27) while such suppression enhances the function of the dNKC (28). AIP in PAS may be associated with changes in energy metabolism at the intracellular level by the defective bioavailability of the insulin-like growth factor (29). This factor domains the transport and uptake of amino acids and glucose in the trophoblast cells and controls the trophoblastic invasion into the decidua through autocrine and paracrine pathways (29). According to Malavolta et al, dietary Zn deficiency causes decreased circulatory levels and inhibits the metabolic action of insulin-like growth factor (30).

Finally, and in relation to the recent theory regarding the genomic alteration of the trophoblasts of PAS, Tzadikevitch et al found that specimens with PAS shortened telomeres (31). These nucleoproteins are found at the chromosomal ends to prevent them from pursuing coalition and degeneration. When they become shorter, cells acquire an arrest mutation during the cell cycle and thus exhibit genomic instability $(31,32)$. Some researchers have reported a direct relationship between the $\mathrm{Zn}$ level and telomere length $(33,34)$. In a study to improve agerelated effects on cells, Farahzadi et al concluded that the use of $\mathrm{Zn}$ supplementation significantly enhanced the length of telomeres (35).

The findings of this study revealed that among women with one previous CS and placenta previa, the maternal serum $\mathrm{Zn}$ level was significantly lower in those with placenta accrete in comparison with those with previa without accrete. A serum $\mathrm{Zn}$ level at $68 \mu \mathrm{g} / \mathrm{dL}$ and below can be used to detect the presence of placenta accrete with comparable accuracy to that of color Doppler US and higher specificity, implying that serum Zn may offer support as a screening tool in the exclusion of placenta accrete in a high-risk population. The accuracy, sensitivity, and specificity of Doppler US in this study were $89.3 \%, 88.9 \%$, and $89.6 \%$, respectively, which disagree with the results of Riteau et al (36). They performed a retrospective study that involved testing the accuracy of both Doppler US and magnetic resonance imaging in which the sensitivity rate of Doppler US was $100 \%$ and the specificity rate was $37.5 \%$. On the contrary, Bowman et al 
(37) conducted a study in which six expert investigators randomly examined the US (some of which were with Doppler) reports of 56 patients with PAS retrospectively over 10 years while they were blinded to the patient's history. The results demonstrated that the sensitivity rate of the US was around $53.5 \%$ and the specificity rate was $88 \%$ while the accuracy was only $64.8 \%$ (37).

The present study attempted to shed light on the risk factors and management options of PAS in comparison with placenta previa without accrete. Silver et al found that the risk of PAS is directly proportional to the number of CS operations. They further reported that the incidence of placenta accrete with one CS was $0.03-3 \%$, increasing to 0.8-61\% with the fourth CS (38). The selection criteria of this study included those with one previous CS in order to decrease the effect of CS repetition on the incidence of PAS. Moreover, patients in this study were matched in terms of age and parity. Thus, unlike most other studies $(7,23,29)$, these factors were not associated with PAS in this study.

All women with PAS in this study ended undergoing hysterectomy either initially or following failed conservative management efforts. Therefore, histopathological specimens were available, AND the presence of the accreta type was the most common observation. Additionally, all kinds of the reported postoperative complications appeared to a higher degree in the PAS group, which is in line with the outcomes of other similar investigations $(21,23,29,39)$. The maternal mortality rate among the PAS group was $5.6 \%$ while Guleria et al (40) reported a maternal mortality rate as high as $22 \%$, attributing it to a delay in arrival, inadequate supplies for blood transfusion, and limited facilities for intensive care in the hospital setting.

\section{Conclusions}

In conclusion, maternal serum $\mathrm{Zn}$ might be a new biomarker for enhancing the use of Doppler US in the antenatal diagnosis of PAS among those with placenta previa or could be used as an inexpensive screening test in situations where color Doppler is unavailable, especially in areas with poor resources. Further evaluations are needed to support the validity of this new hypothesis.

\section{Authors' Contribution}

ZAJ Designed, did data collection and statistical analysis. RZO participated in Writing and editing the manuscript. DAS designed and did data collection.

\section{Conflict of Interests}

Authors declare that they have no conflict of interests.

\section{Ethical Issues}

The study protocol was approved by the Ethics Committee of the Obstetrical Department, Medical College, Al-Mustansiriyah University, Iraq. Verbally informed consent was taken from the participants.

\section{Financial Support}

The work was funded by the authors.

\section{Acknowledgments}

We express our gratitude to all pregnant ladies who took part in the study.

\section{References}

1. Cahill AG, Beigi R, Heine RP, Silver RM, Wax JR. Placenta accreta spectrum. Am J Obstet Gynecol. 2018;219(6):B2-B16. doi:10.1016/j.ajog.2018.09.042

2. Jauniaux E, Collins S, Burton G). Placenta accreta spectrum: pathophysiology and evidence-based anatomy for prenatal ultrasound imaging. Am J Obstet Gynecol. 2018;218(1):75-87. doi:10.1016/j.ajog.2017.05.067

3. Jauniaux E, Ayres-de-Campos D. FIGO consensus guidelines on placenta accreta spectrum disorders: introduction. Int J Gynaecol Obstet. 2018;140(3):261-264. doi:10.1002/ijgo.12406

4. Booker W, Moroz L. Abnormal placentation. Semin Perinatol. 2019;43(1):51-59. doi:10.1053/j.semperi.2018.11.009

5. Royal College of Obstetricians and Gynaecologists (RCOG). Placenta Praevia, Placenta Praevia Accreta and Vasa Praevia: Diagnosis and Management. London: RCOG; 2011:1-26.

6. Thompson O, Otigbah C, Nnochiri A, Sumithran E, Spencer K. First trimester maternal serum biochemical markers of aneuploidy in pregnancies with abnormally invasive placentation. BJOG. 2015;122(10):1370-1376. doi:10.1111/1471-0528.13298

7. Lyell DJ, Faucett AM, Baer RJ, et al. Maternal serum markers, characteristics and morbidly adherent placenta in women with previa. J Perinatol. 2015;35(8):570-574. doi:10.1038/jp.2015.40

8. Bartels HC, Postle JD, Downey P, Brennan DJ. Placenta accreta spectrum: a review of pathology, molecularbiology, and biomarkers. Dis Markers. 2018;2018:1507674. doi:10.1155/2018/1507674

9. Adami R, Fratto VM, Srinivasan S, et al. Discovery of maternal serum miRNA biomarkers for detection of placenta accreta. Am J Obstet Gynecol. 2019;220(1):S394-S395. doi:10.1016/j. ajog.2018.11.617

10. Wang $\mathrm{H}, \mathrm{Hu} \mathrm{YF}, \mathrm{Hao} \mathrm{JH}$, et al. Maternal zinc deficiency during pregnancy elevates the risks of fetal growth restriction: a population-based birth cohort study. Sci Rep. 2015;5:11262. doi:10.1038/srep11262

11. Jaafar ZAA, Salman DA, Obeid RZ. The role of maternal and fetal serum zinc level in low birth weight. J Pharm Sci Res. 2018;10(8):2115-2118.

12. Ofakunrin A, Collins J, Diala U, Afolaranmi T, Okolo S. Relationship between maternal serum zinc, cord blood zinc and birth weight of term newborn infants in Jos, Plateau State, Nigeria. Jos Journal of Medicine. 2017;11(2):12-20.

13. Al-Jameil N, Tabassum H, Ali MN, Qadeer MA, Khan FA, AlRashed M. Correlation between serum trace elements and risk of preeclampsia: a case controlled study in Riyadh, Saudi Arabia. Saudi J Biol Sci. 2017;24(6):1142-1148. doi:10.1016/j. sjbs.2015.02.009

14. MaY, Shen $X$, Zhang D. The relationship between serum zinc level and preeclampsia: a meta-analysis. Nutrients. 2015;7(9):78067820. doi:10.3390/nu7095366

15. Kucukaydin Z, Kurdoglu M, Kurdoglu Z, Demir H, Yoruk $\mathbf{I H}$. Selected maternal, fetal and placental trace element and heavy metal and maternal vitamin levels in preterm deliveries with or without preterm premature rupture of membranes. J Obstet Gynaecol Res. 2018;44(5):880-889. doi:10.1111/jog.13591

16. Ferretti C, Bruni L, Dangles-Marie V, Pecking AP, Bellet D. Molecular circuits shared by placental and cancer cells, and their implications in the proliferative, invasive and migratory capacities of trophoblasts. Hum Reprod Update. 2007;13(2):121-141. doi:10.1093/humupd/dml048

17. Yagel S, Parhar RS, Jeffrey JJ, Lala PK. Normal nonmetastatic human trophoblast cells share in vitro invasive properties of malignant cells. J Cell Physiol. 1988;136(3):455-462. doi:10.1002/ jcp.1041360309

18. Ho E. Zinc deficiency, DNA damage and cancer risk.J Nutr Biochem. 2004;15(10):572-578. doi:10.1016/j.jnutbio.2004.07.005 
19. Zowczak M, Iskra M, Torliński L, Cofta S. Analysis of serum copper and zinc concentrations in cancer patients. Biol Trace Elem Res. 2001;82(1-3):1-8. doi:10.1385/bter:82:1-3:001

20. Guerado E, Medina A, Mata MI, Galvan JM, Bertrand ML. Protocols for massive blood transfusion: when and why, and potential complications. Eur J Trauma Emerg Surg. 2016;42(3):283-295. doi:10.1007/s00068-015-0612-y

21. Büke B, Akkaya H, Demir S, et al. Relationship between first trimester aneuploidy screening test serum analytes and placenta accreta. J Matern Fetal Neonatal Med. 2018;31(1):59-62. doi:10.1 080/14767058.2016.1275546

22. Desai N, Krantz D, Roman A, Fleischer A, Boulis S, Rochelson B. Elevated first trimester PAPP--a is associated with increased risk of placenta accreta. Prenat Diagn. 2014;34(2):159-162. doi:10.1002/pd.4277

23. Oztas E, Ozler S, Caglar AT, Yucel A. Analysis of first and second trimester maternal serum analytes for the prediction of morbidly adherent placenta requiring hysterectomy. Kaohsiung J Med Sci. 2016;32(11):579-585. doi:10.1016/j.kjms.2016.08.011

24. Tseng J, Chou MM. Differential expression of growth-, angiogenesis- and invasion-related factors in the development of placenta accreta. Taiwan J Obstet Gynecol. 2006;45(2):100-106. doi:10.1016/s1028-4559(09)60205-9

25. Hoseini Z, Azimi-Nezhad M, Ghayour-Mobarhan M, et al. VEGF gene polymorphism interactions with dietary trace elements intake in determining the risk of metabolic syndrome. J Cell Biochem. 2018. doi:10.1002/jcb.27171

26. Jabrane-Ferrat $\mathrm{N}$, Siewiera J. The up side of decidual natural killer cells: new developments in immunology of pregnancy. Immunology. 2014;141(4):490-497. doi:10.1111/imm.12218

27. Maywald M, Rink L. Zinc supplementation induces CD4(+) CD25(+)Foxp3(+) antigen-specific regulatory $T$ cells and suppresses IFN- $\gamma$ production by upregulation of Foxp3 and KLF-10 and downregulation of IRF-1. Eur J Nutr. 2017;56(5):1859-1869. doi:10.1007/s00394-016-1228-7

28. Rolles B, Maywald M, Rink L. Influence of zinc deficiency and supplementation on NK cell cytotoxicity. J Funct Foods. 2018;48:322-328. doi:10.1016/j.jff.2018.07.027

29. Kilcoyne A, Shenoy-Bhangle AS, Roberts DJ, Sisodia RC, Gervais DA, Lee SI. MRI of placenta accreta, placenta increta, and placenta percreta: pearls and pitfalls. AJR Am J Roentgenol. 2017;208(1):214-221. doi:10.2214/ajr.16.16281

30. Malavolta M, Piacenza F, Costarelli L, et al. Zinc, insulin and IGF-I
Interplay in aging. In: Rattan S, Sharma R, eds. Hormones in Ageing and Longevity. Cham: Springer; 2017:57-90. doi:10.1007/978-3319-63001-4_4

31. Tzadikevitch Geffen K, Gal H, Vainer I, et al. Senescence and telomere homeostasis might be involved in placenta percretapreliminary investigation. Reprod Sci. 2018;25(8):1254-1260. doi:10.1177/1933719117737852

32. Armanios M. Telomeres and age-related disease: how telomere biology informs clinical paradigms. J Clin Invest. 2013;123(3):9961002. doi:10.1172/jci66370

33. Cipriano C, Tesei S, Malavolta M, et al. Accumulation of cells with short telomeres is associated with impaired zinc homeostasis and inflammation in old hypertensive participants. J Gerontol A Biol Sci Med Sci. 2009;64(7):745-751. doi:10.1093/gerona/glp048

34. Ojeda-Rodriguez A, Morell-Azanza L, Alonso-Pedrero L, del Moral AM. Aging, telomere integrity, and antioxidant food. In: del Moral AM, Aguilera García CM, eds. Obesity. Academic Press; 2018:241-261. doi:10.1016/b978-0-12-812504-5.00012-x

35. Farahzadi R, Fathi E, Mesbah-Namin SA, Zarghami N. Zinc sulfate contributes to promote telomere length extension via increasing telomerase gene expression, telomerase activity and change in the TERT gene promoter CpG island methylation status of human adipose-derived mesenchymal stem cells. PLoS One. 2017;12(11):e0188052. doi:10.1371/journal.pone.0188052

36. Riteau AS, Tassin M, Chambon G, et al. Accuracy of ultrasonography and magnetic resonance imaging in the diagnosis of placenta accreta. PLoS One. 2014;9(4):e94866. doi:10.1371/ journal.pone.0094866

37. Bowman ZS, Eller AG, Kennedy AM, et al. Accuracy of ultrasound for the prediction of placenta accreta. Am J Obstet Gynecol. 2014;211(2):177.e171-177. doi:10.1016/j.ajog.2014.03.029

38. Clark EA, Silver RM. Long-term maternal morbidity associated with repeat cesarean delivery. Am J Obstet Gynecol. 2011;205(6 Suppl):S2-10. doi:10.1016/j.ajog.2011.09.028

39. Alchalabi H, Lataifeh I, Obeidat B, Zayed F, Khader YS, Obeidat N. Morbidly adherent placenta previa in current practice: prediction and maternal morbidity in a series of 23 women who underwent hysterectomy. J Matern Fetal Neonatal Med. 2014;27(17):17341737. doi:10.3109/14767058.2013.879700

40. Guleria K, Gupta B, Agarwal S, Suneja A, Vaid N, Jain S. Abnormally invasive placenta: changing trends in diagnosis and management. Acta Obstet Gynecol Scand. 2013;92(4):461-464. doi:10.1111/aogs.12083

(C) 2021 The Author(s); This is an open-access article distributed under the terms of the Creative Commons Attribution License (http:// creativecommons.org/licenses/by/4.0), which permits unrestricted use, distribution, and reproduction in any medium, provided the original work is properly cited. 